\title{
Increasing gaps in health inequalities related to non-communicable diseases in South Australia; implications towards behavioural risk factor surveillance systems to provide evidence for action
}

\author{
Stefano Campostrini ${ }^{1,2^{*}}$, Eleonora Dal Grande ${ }^{3}$ and Anne W. Taylor ${ }^{3}$ (D)
}

\begin{abstract}
Background: Although Australia is a country cited as having generally low health inequalities among different socioeconomic groups, inequalities have persisted. The aim of this analysis was to highlight how inequalities have evolved over a 13 years period in South Australia (SA).

Methods: Since 2002, over 600 interviews per month have been undertaken with SA residents through a computer assisted telephone survey method (total 77,000+). Major risk factors and chronic diseases have been analyzed providing trends by two socio-economic variables: education and a proxy of income (ability to save).

Results: While income and educational gaps are reducing over time in SA, those that remain in the lower socioeconomic groups have a generally higher prevalence of risk factors and chronic diseases. The health disparity gap is still relevant, although at a different extent, for all the variables considered in our study, with most appearing to be stable if not increasing over time.
\end{abstract}

Conclusions: Surveillance can be a good source of information both to show the evolution of problems and to evaluate possible future interventions. Extensive effort is still required to "close the gap" of health inequalities in SA. More precisely targeted and properly implemented interventions are needed.

Keywords: Behavioral risk factor surveillance, Chronic diseases, Non communicable diseases, Health inequalities, Trend analysis

\section{Background}

Australia is a country cited as having low health inequalities [1]. In 1994, health authorities in Australia published a report setting targets for better health outcomes [2]. The report highlighted the importance of monitoring different health outcomes between different socio-economic and demographic groups [2]. Although considerable attention has been paid to inequalities since the first national reports, and many calls for action have been made, health

\footnotetext{
* Correspondence: stefano.campostrini@unive.it

${ }^{1}$ Department of Economics, Ca' Foscari University of Venice, San Giobbe 873, 30121 Venice, Italy

${ }^{2}$ School of Medicine, The University of Adelaide, Adelaide, South Australia Full list of author information is available at the end of the article
}

inequalities have persisted among different socioeconomic groups [3-5]. More recently, several interventions have been promoted, both at a public health and other government agency level. Notably in 2010, a document 'Health in all Policies', cited explicitly as a tool to tackle health disparities has been produced conjointly by the World Health Organization (WHO) and the Government of SA [6].

Although some positives changes have occurred in SA, Australia and worldwide (eg smoking), other targets are yet to be reached [5-7]. Relevant chronic disease and risk factor targets that require constant monitoring and assessment include harmful use of alcohol, physical inactivity, salt intake, tobacco use, diabetes and obesity [8].

C The Author(s). 2019 Open Access This article is distributed under the terms of the Creative Commons Attribution 4.0 International License (http://creativecommons.org/licenses/by/4.0/), which permits unrestricted use, distribution, and reproduction in any medium, provided you give appropriate credit to the original author(s) and the source, provide a link to the Creative Commons license, and indicate if changes were made. The Creative Commons Public Domain Dedication waiver (http://creativecommons.org/publicdomain/zero/1.0/) applies to the data made available in this article, unless otherwise stated. 
The use of a behavioural risk factor surveillance (BRFS) system as a valuable source of information for inequalities monitoring has been advocated, particularly in relation to tracking trends $[9,10]$. While some of the targets are not possible to monitor with the use of a BRFS system, others are. As such, we analyzed data from the South Australian Monitoring and Surveillance System (SAMSS) to test the most recent relevant trends on specific health disparities. The focus of these first analyses were to focus solely on trends, without at this stage, investigating possible causes, leaving this to future studies.

\section{Methods}

Data

SAMSS is owned by the South Australian Department of Health and Ageing (SA Health) and is an epidemiological monitoring system. SAMSS aims to detect and facilitate understanding of trends in the prevalence of chronic conditions, risk and protective factors, and other determinants of health. These data monitor departmental, state and national priority areas and are linked to key indicators such as state and national healthy weight targets [11].

Each month from July 2002, a sample of South Australians was randomly selected from the Electronic White Pages (EWP). Introductory letters were sent to each household selected to inform them of the upcoming telephone survey, and inviting the person who had the last birthday in the household to participate in a telephone interview. The interviews were conducted by professional interviewers, using Computer Assisted Telephone Interview (CATI) technology. Approximately 600 respondents participate in each SAMSS survey. Although data were collected on children, data presented in these analyses are for those aged 18 years and older. All data are weighted each month by sex, age, area of residence and probability of selection of the household using the latest Australian Bureau of Statistics (ABS) census data or estimated residential population data. Data were then raked to further adjust for weighting [12]. Data from July 2002 until June 2015 were utilised. Ethics approval for the survey was obtained from the ethics committee of the SA Health (HREC/14/SAH/ 200 \& HREC/436.02.2014). The topics and questions included in SAMSS were developed in consultation with key personnel within SA Health, including relevant experts, and questions are based on previous work undertaken in Australian states and territories. Where possible, questions that had previously been included in other surveys, and which were perceived to ascertain reliable and valid data, were used or modified. Additional details on SAMSS methodology is available [11].

\section{Variables}

The risk factors assessed were: overweight/obesity (calculated from self-reported height and weight and recoded into Body Mass Index (BMI) according to the WHO classification with $\mathrm{BMI} \geq 25$ nominated as unhealthy and $\mathrm{BMI} \geq 30$ classified as obesity) and fruit consumption (two or more serves per day, as suggested by National Health \& Medical Research Council [13] and international guidelines [14].

We also examined the self-reported prevalence of chronic conditions, defined by answers to the question "have you ever been told by a doctor that you have... diabetes, asthma, heart disease, osteoporosis and/or arthritis". Having any chronic condition (one or more) and multimorbidity (two or more) of these conditions were used in the analysis. Specific analyses were also undertaken for diabetes which is acknowledged as one of the chronic condition more sensitive to social determinants of health $[15,16]$.

Finally, we analysed self-reported mental health conditions, a combined variable created using positive answers to the question "Have you been told by a doctor that you have any of the following conditions (yes/no) in the last 12 months? The conditions were: anxiety, depression, stress related problems or any other mental health problem" and/or whether the respondent was currently receiving treatment for these conditions. Psychological distress was also measured by the Kessler 10 (K10) [17-19]. The K10 is a self-report, 10-item set of questions based on the level of anxiety and depressive symptoms experienced in the previous 4 weeks. The questions were scored to a single scaled item with respondents with high scores of 22-50 being classified as having high or very high levels of psychological distress.

To show possible disparities, we used the following two demographic variables: education (no school to secondary, trade certificate or diploma, degree or higher) and a question assessing "which best describes your money situation" (we spend more money than we get; we have just enough to get through to the next pay day; there's money left over but we just spend it; we can save a bit now and then; we can save a lot) with the last two categories recoded as 'able to save'. This question was used rather than household income as income has increased over this time period (in line with Consumer Price Index (CPI) increases).

\section{Statistical analysis}

Analyses were conducted using chi-squared test for trend to detect differences in overall prevalence and for each level of educational attainment and household money situation for each risk factor and chronic condition between 2002/4 and 2013/15. Chi-squared tests were also undertaken to detect differences in prevalence of each risk factor and chronic condition by educational attainment and 'ability to save' for 2002/4 and 2013/15. All "don't know" responses were treated as missing 
values. Annual prevalence over time for each of the risk factors and chronic conditions by educational attainment and 'ability to save' are presented graphically. The prevalence over time data were not standardized to a reference population. In total $n=74,127$ interviews were conducted with adults aged 18 years or older. Response rates (RR) using the American association for Public Opinion Research [20] standards definition (AAPOR RR1) varied from 54.1 to $71.3 \%$ (mean $=65.5 \%$ ).

Data were analysed using SPSS Version 20.0 (IBM SPSS Statistics, Armonk, NY, USA) and Stata (StataCorp, College Station, TX, USA) Version 13.0. All data presented were weighted to be reflective of the South Australian population using raking methodology by area (metropolitan/rural), age, gender, marital status, country of birth, educational attainment, and dwelling status (rented property vs other) to the most relevant South Australian population data (year), and probability of selection in the household.

\section{Results}

The total number of interviews and response rate per year are included as Additional file 1: Table S1. In SA over the past 13 years the population have achieved higher levels of education (related to the fact that younger generations have generally higher level of education), with the proportion achieving a university degree or higher increasing from $12.6 \%$ (95\% CI 12.0-13.2) to 18.7\% (95\% CI 18.2-19.3). In addition, the South Australian population are relatively richer with the ability to save increasing from $63.0 \%$ (95\% CI $62.2-63.9)$ to $66.6 \%$ (95\% CI 65.9-67.3) (Table 1).

The subjectively measured income related figures are confirmed by those of the ABS that has generally reported a steady increase in the mean real equalized disposable household income over this period (with the only exception of years around 2010 in which no increase was observed) [21]. As this increase was across all the income quartiles, income disparity does not seem to have increased in the last decade in SA.

The analysis shows substantial differences in the prevalence of risk factors and chronic diseases among socio-economic subgroups (Table 1 and Figs. 1, 2, 3, 4, $5,6,7,8)$. In regard to unhealthy weight, the overall increase over time is greater among the lower educated groups $(P<0.001)$, with a smaller increase seen for those with a university education. Similar results were seen for obesity, although there were increases across all social groups with the gap increasing over time.

In regard to fruit consumption, the higher educated group have changed little over the years, while in the other groups increases in consumption were apparent although starting from a lower base. There was an increase in fruit consumption for those who could save but lower estimates and no increase over time for those who cannot save, confirming a substantial inequality gap also for this variable.

The prevalence of diabetes, as expected, demonstrates differences among the lower social economic subgroups $(P<0.001)$ with the only subgroup that has not seen an increase over time being the higher educated group. Prevalence estimates continue to be lower for the higher social economic groups, and the gap in the other groups is increasing.

In regard to mental health conditions, the gap associated with education level increased over time, with the higher educated groups relatively stable while the other groups increased in prevalence $(P<0.001)$. In terms of psychological distress, the overall level decreased by $2 \%$ over time $(P<0.001)$ as did both the lowest and highest educated groups. Only a small decrease was shown among the 'unable to save' group, but this group is still far from closing a substantial gap in prevalence: $18 \%$ for the 'unable to save' group compared to $7 \%$ for the 'can save' group.

Differences in the prevalence of those with one chronic condition were relatively stable over time, with the expected (as highlighted by the other variables above) differences among socioeconomic groups. The differences are much more relevant when we consider those with two or more chronic conditions. The gap, again increasing over time, occurs as a result of the lower educated and those with lower economic conditions changing for the worse $(P=0.001$ and $P<0.001)$ while the other groups are substantially stable over time.

\section{Discussion}

SAMSS data have shown that health inequalities are stable, if not increasing, in SA with health disparities for lower educated and lower income groups, measured by those unable to save, appearing to be increasing in most of the health variables considered. Although it is not an aim of this manuscript to speculate about possible causes, which are quite often difficult to define [22], it is conceivable that most of these increases in inequality can be related to interventions not targeted to specific groups, or not specifically designed to be capable of reducing the gap [23-25]. Interventions targeted to the general population [23], such as, e.g., building cycling tracks (now covering most of the South Australian urban area), could be of benefit for those already active (typically higher educated, with higher income) and have no impact on those more vulnerable that are unable to buy a bike. This eventually produces an increase in the gap in the level of sedentary activity between classes. A call for more action and better understanding for more effectively targeting of the interventions is warranted. Staying with the biking example, this would mean health 
Table 1 Socio-demographic situation, risk factors, chronic diseases and mental health comparison

\begin{tabular}{|c|c|c|c|c|c|c|c|}
\hline & \multicolumn{3}{|c|}{$2002 / 4$} & \multicolumn{3}{|c|}{$2013 / 15$} & \multirow{2}{*}{$\begin{array}{l}\text { Cmp 2002/4 and 2013/15 } \\
P_{\text {value }}\end{array}$} \\
\hline & $\mathrm{n}$ & $\%(95 \% \mathrm{Cl})$ & $P$ value ${ }^{a}$ & $\mathrm{n}$ & $\%(95 \% \mathrm{Cl})$ & $P$ value $^{b}$ & \\
\hline \multicolumn{8}{|l|}{ Demograpic Variables } \\
\hline \multicolumn{8}{|l|}{ Educational attainment } \\
\hline No schooling to secondary & 7932 & $62.8(62.0-63.6)$ & & 7969 & $47.7(47.0-48.5)$ & & $<0.001$ \\
\hline Trade, certificate, diploma & 3112 & $24.6(23.9-25.4)$ & & 5602 & $33.5(32.8-34.3)$ & & \\
\hline Degree or higher & 1587 & $12.6(12.0-13.2)$ & & 3132 & $18.7(18.2-19.3)$ & & \\
\hline \multicolumn{8}{|l|}{ Household money situation } \\
\hline Unable to save & 4238 & $33.5(32.7-34.3)$ & & 4654 & $27.8(27.1-28.5)$ & & $<0.001$ \\
\hline Can save & 7974 & $63.0(62.2-63.9)$ & & 11,152 & $66.6(65.9-67.3)$ & & \\
\hline Not stated & 438 & $3.5(3.2-3.8) *$ & & 930 & $5.6(5.2-5.9)$ & & \\
\hline \multicolumn{8}{|l|}{ Risk Factors } \\
\hline Overweight/Obesity & 6487 & $54.9(54.0-55.8)$ & & 9570 & $60.8(60.1-61.6)$ & & $<0.001$ \\
\hline No schooling to secondary & 4104 & $56.1(55.0-57.2)$ & $<0.001$ & 4569 & $61.9(60.8-63.0)$ & $<0.001$ & $<0.001$ \\
\hline Trade, certificate, diploma & 1655 & $55.9(54.1-57.7)$ & & 3445 & $65.0(63.7-66.3)$ & & $<0.001$ \\
\hline Degree or higher & 720 & $47.5(45.0-50.0)$ & & 1548 & $51.0(49.2-52.8)$ & & 0.03 \\
\hline Unable to save & 2280 & $58.3(56.8-59.9)$ & $<0.001$ & 2764 & $63.2(61.8-64.7)$ & .001 & $<0.001$ \\
\hline Can save & 4033 & $53.6(52.5-54.8)$ & & 6383 & $60.4(59.5-61.4)$ & & $<0.001$ \\
\hline Obese & 2270 & $19.2(18.5-20.0)$ & & 4165 & $26.5(25.8-27.2)$ & & $<0.001$ \\
\hline No schooling to secondary & 1516 & $20.7(19.8-21.7)$ & $<0.001$ & 2085 & $28.2(27.2-29.3)$ & $<0.001$ & $<0.001$ \\
\hline Trade, certificate, diploma & 555 & $18.8(17.4-20.2)$ & & 1521 & $28.7(27.5-30.0)$ & & $<0.001$ \\
\hline Degree or higher & 198 & $13.1(11.5-14.9)$ & & 557 & $18.3(17.0-19.8)$ & & $<0.001$ \\
\hline Unable to save & 911 & $23.3(22.0-24.7)$ & $<0.001$ & 1370 & $31.3(30.0-32.7)$ & $<0.001$ & $<0.001$ \\
\hline Can save & 1300 & $17.3(16.5-18.2)$ & & 2626 & $24.9(24.1-25.7)$ & & $<0.001$ \\
\hline Sufficient fruit consumption & 5026 & $39.7(38.9-40.6)$ & & 7062 & $42.3(41.6-43.1)$ & & $<0.001$ \\
\hline No schooling to secondary & 3070 & $38.7(37.6-39.8)$ & $<0.001$ & 3224 & $40.6(39.5-41.7)$ & $<0.001$ & 0.02 \\
\hline Trade, certificate, diploma & 1202 & $38.6(36.9-40.3)$ & & 2302 & $41.1(39.9-42.4)$ & & 0.02 \\
\hline Degree or higher & 744 & $46.9(44.4-49.4)$ & & 1526 & $48.7(47.0-50.5)$ & & 0.23 \\
\hline Unable to save & 1466 & $34.6(33.2-36.0)$ & $<0.001$ & 1653 & $35.6(34.3-37.0)$ & $<0.001$ & 0.31 \\
\hline Can save & 3386 & $42.5(41.4-43.6)$ & & 5024 & $45.1(44.2-46.0)$ & & $<0.001$ \\
\hline \multicolumn{8}{|l|}{ Chronic Conditions } \\
\hline Diabetes & 875 & $6.9(6.5-7.4)$ & & 1516 & $9.1(8.6-9.5)$ & & $<0.001$ \\
\hline No schooling to secondary & 609 & $7.7(7.1-8.3)$ & $<0.001$ & 822 & $10.3(9.7-11.0)$ & $<0.001$ & $<0.001$ \\
\hline Trade, certificate, diploma & 184 & $5.9(5.1-6.8)$ & & 507 & $9.1(8.3-9.8)$ & & $<0.001$ \\
\hline Degree or higher & 80 & $5.1(4.1-6.2)$ & & 184 & $5.9(5.1-6.7)$ & & 0.25 \\
\hline Unable to save & 343 & $8.1(7.3-9.0)$ & $<0.001$ & 524 & $11.3(10.4-12.2)$ & $<0.001$ & $<0.001$ \\
\hline Can save & 513 & $6.4(5.9-7.0)$ & & 916 & $8.2(7.7-8.7)$ & & $<0.001$ \\
\hline Current mental health condition & 1987 & $15.7(15.1-16.4)$ & & 3364 & $20.1(19.5-20.7)$ & & $<0.001$ \\
\hline No schooling to secondary & 1240 & $15.6(14.8-16.4)$ & 0.74 & 1659 & $20.8(19.9-21.7)$ & $<0.001$ & $<0.001$ \\
\hline Trade, certificate, diploma & 501 & $16.1(14.9-17.4)$ & & 1209 & $21.6(20.5-22.7)$ & & $<0.001$ \\
\hline Degree or higher & 243 & $15.3(13.6-17.2)$ & & 494 & $15.8(14.5-17.1)$ & & 0.68 \\
\hline Unable to save & 1007 & $23.8(22.5-25.1)$ & $<0.001$ & 1411 & $30.3(29.0-31.7)$ & $<0.001$ & $<0.001$ \\
\hline Can save & 918 & $11.5(10.8-12.2)$ & $<0.001$ & 1790 & $16.0(15.4-16.7)$ & & $<0.001$ \\
\hline Psychological distress & 1533 & $12.1(11.5-12.7)$ & & 1678 & $10.1(9.6-10.6)$ & & $<0.001$ \\
\hline No schooling to secondary & 1058 & $13.3(12.6-14.1)$ & $<0.001$ & 841 & $10.7(10.0-11.4)$ & $<0.001$ & $<0.001$ \\
\hline
\end{tabular}


Table 1 Socio-demographic situation, risk factors, chronic diseases and mental health comparison (Continued)

\begin{tabular}{|c|c|c|c|c|c|c|c|}
\hline & \multicolumn{3}{|c|}{$2002 / 4$} & \multicolumn{3}{|c|}{$2013 / 15$} & \multirow{2}{*}{$\begin{array}{l}\text { Cmp 2002/4 and 2013/15 } \\
P_{\text {value }}\end{array}$} \\
\hline & $\mathrm{n}$ & $\%(95 \%$ Cl) & $P$ value $^{a}$ & $\mathrm{n}$ & $\%(95 \% \mathrm{Cl})$ & $P$ value $^{b}$ & \\
\hline Trade, certificate, diploma & 318 & $10.2(9.2-11.3)$ & & 633 & $11.3(10.5-12.2)$ & & 0.11 \\
\hline Degree or higher & 152 & $9.6(8.2-11.1)$ & & 203 & $6.5(5.7-7.4)$ & & $<0.001$ \\
\hline Unable to save & 876 & $20.7(19.5-21.9)$ & $<0.001$ & 826 & $17.9(16.8-19.0)$ & $<0.001$ & 0.001 \\
\hline Can save & 607 & $7.6(7.0-8.2)$ & & 778 & $7.0(6.5-7.5)$ & & 0.11 \\
\hline At least one Chronic Condition & 5063 & $40.0(39.2-40.9)$ & & 6664 & $39.8(39.1-40.6)$ & & 0.73 \\
\hline No schooling to secondary & 3407 & $42.9(41.9-44.0)$ & $<0.001$ & 3537 & $44.4(43.3-45.5)$ & $<0.001$ & 0.07 \\
\hline Trade, certificate, diploma & 1175 & $37.8(36.1-39.5)$ & & 2194 & $39.2(37.9-40.4)$ & & 0.20 \\
\hline Degree or higher & 471 & $29.7(27.5-32.0)$ & & 924 & $29.5(27.9-31.1)$ & & 0.91 \\
\hline Unable to save & 1891 & $44.6(43.1-46.1)$ & $<0.001$ & 2133 & $45.8(44.4-47.3)$ & $<0.001$ & 0.26 \\
\hline Can save & 3025 & $37.9(36.9-39.0)$ & & 4189 & 37.6 (36.7-38.5) & & 0.60 \\
\hline Two or more Chronic Conditions & 1538 & $12.2(11.6-12.8)$ & & 2172 & $13.0(12.4-13.6)$ & & 0.04 \\
\hline No schooling to secondary & 1111 & $14.0(13.3-14.8)$ & $<0.001$ & 1326 & $16.6(15.8-17.5)$ & $<0.001$ & 0.001 \\
\hline Trade, certificate, diploma & 320 & $10.3(9.3-11.4)$ & & 639 & $11.4(10.6-12.3)$ & & 0.11 \\
\hline Degree or higher & 104 & $6.5(5.4-7.9)$ & & 204 & $6.5(5.7-7.4)$ & & 0.98 \\
\hline Unable to save & 614 & $14.5(13.5-15.6)$ & $<0.001$ & 815 & $17.5(16.5-18.6)$ & $<0.001$ & $<0.001$ \\
\hline Can save & 890 & $11.2(10.5-11.9)$ & & 1240 & $11.1(10.5-11.7)$ & & 0.92 \\
\hline
\end{tabular}

Note $^{a}-\mathrm{p}$ values are associated with chi-squared test of risk factor/chronic condition and educational attainment/household money situation for $2002 / 4$ only; ${ }^{b}: p$ values are associated with chi-squared test of risk factor/chronic condition and educational attainment/household money situation for $2013 / 15$ only; ${ }^{c}$ : $p$ values are associated with chi-squared test for trend between two time points, 2002/4 and 2013/15 for corresponding risk factor/chronic condition, and for each category of educational attainment/household money situation by risk factor/chronic condition

promotion activities involving more vulnerable communities. This could include, for example, offering free bikes, activities aimed to engage people in small bike tours, offering bikes to cycle to school and creating the conditions where this could easily happen.

It is evident, from the analysis presented in this study, the substantial role played by non-communicable disease and behavioural risk factor surveillance systems, in showing the evolutionary aspects of health disparities. Certainly, for these analyses the availability of a 'real' surveillance system $[26,27]$ rather than a few scattered surveys is fundamental. In our opinion, yearly or even with less frequently repeated surveys (the WHO suggest 'at least every five years') [28] provide little information when studying and showing trends. We believe that these analyses have only scratched the surface: much information can still to be obtained from surveillance data, particularly in understanding the mechanisms [29] which create health inequalities and, as we have seen, increase these inequalities. Specific analyses for sub-groups, defined on the basis of socio-demographic variables, but also geographically, can provide further information [30-32]. Data from surveillance systems, highlighted in this study, could be even more useful when linked with data from other sources (e.g. Census data), to study other potentially influencing social determinants such as social and cultural capital [33] or urban settings [34]. An even more important role in the future could have surveillance showing 'what works' in reducing health inequalities (when targeted interventions are implemented), given the potential use of these systems for evaluation purposes [4, 35, 36].

In this first paper we purposely limited the analyses so as to show simple time trends. Research is needed on these data, to better understand interaction of different social determinants of health, and the possible underlying mechanisms which creates and reinforces gaps. Certainly, for instance, the fact that over the years the number of those falling into more deprived groups has decreased in SA, due to the selective effect of social mobility, and could have left individuals in the lower strata individuals with characteristics that (directly or indirectly) induce worse health attitudes and behaviours.

However, using simple analyses to show how much the health inequality gap remains relevant, also creates several limitations. Some of these are related to the available data, and some are associate with the analysis conducted. A first weakness is the limiting of the risk factors assessed to two (BMI and fruit consumption) and three specific chronic conditions (diabetes, mental health and psychological distress). In addition, only two socio-economic related variables were used. Although the use of 'ability to save' as an indicator has been shown in Australia to be a valid indicator of financial security [37], it is acknowledged that other more reliable 

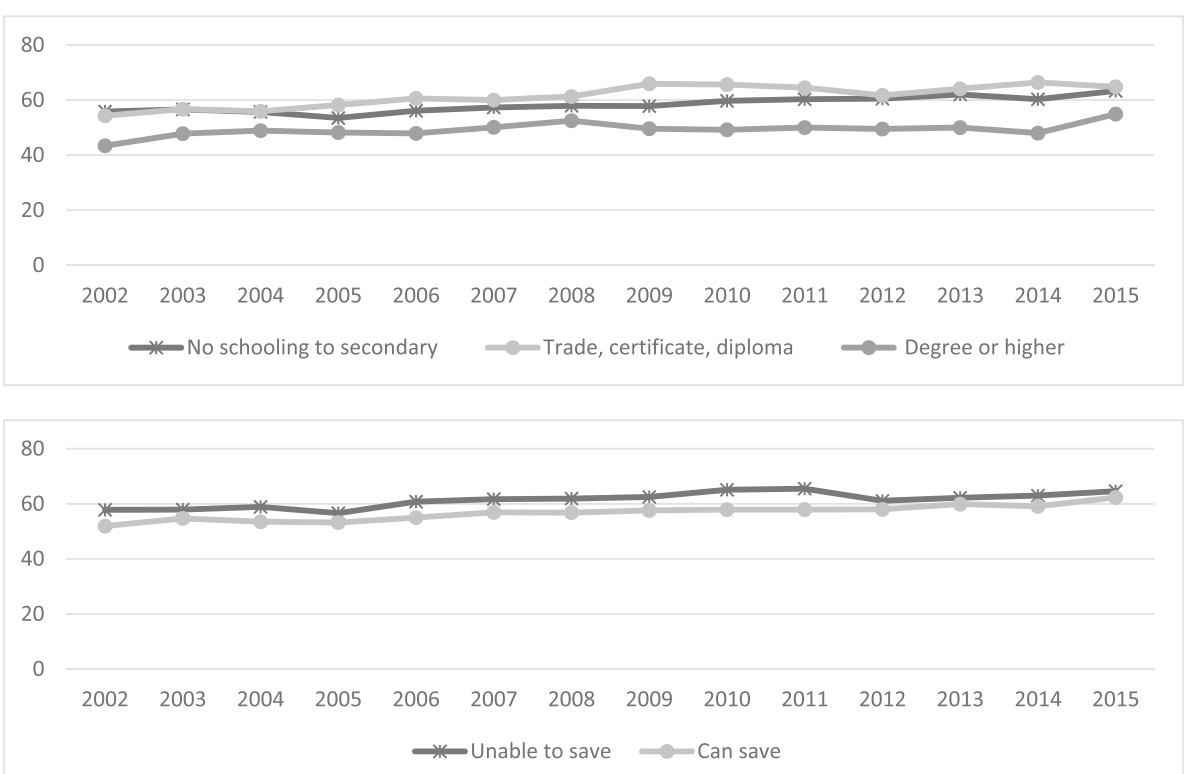

Fig. 1 Annual prevalence, overweight and obesity by educational attainment and household money situation, 2002 to 2015

questions could have been used such as income. However, over the 10 year period, income earnings have increased for the whole of Australia which made it difficult to have comparable income groupings across the years. This study uses self-reported surveys which can potentially be subjected to bias due to socially desirable responses leading to possible over- or under-estimation of behaviors or health conditions, such as having a mental health condition or overweight and obesity due to incorrect reporting of height and weight [38]. However, these biases are of little importance if the aim of SAMSS is to study changes in the behaviour or health condition over time, assuming that the level of bias is constant over time.

The use of listed telephone numbers as the sampling frame can be considered a weakness of this study due to

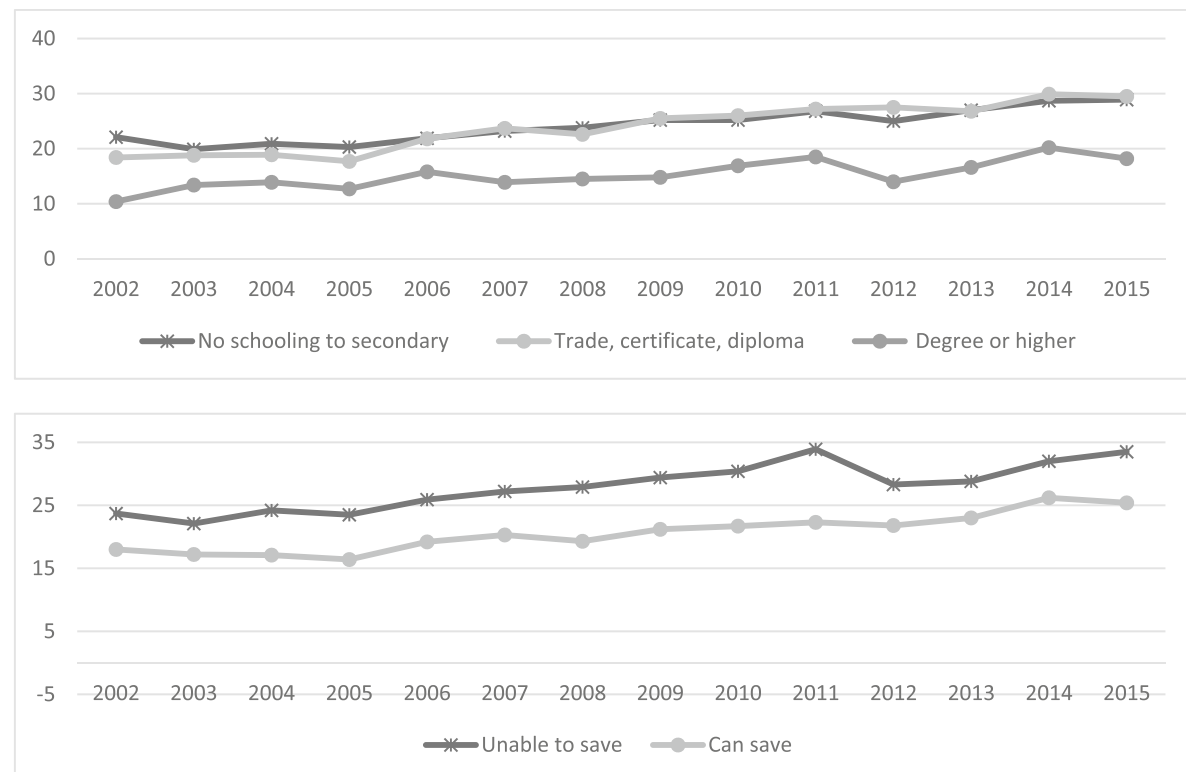

Fig. 2 Annual prevalence, obese by educational attainment and household money situation, 2002 to 2015 

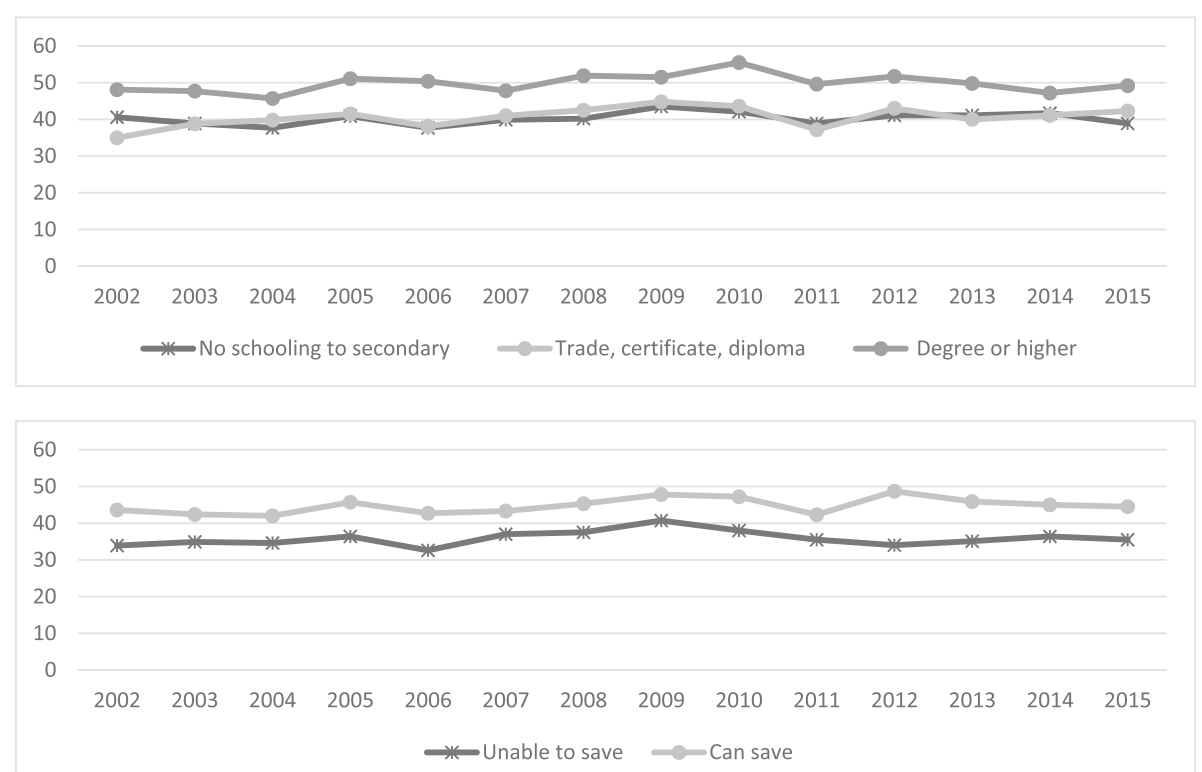

Fig. 3 Annual prevalence, consuming sufficient amount of fruit daily ( 2 or more serves) by educational attainment and household money situation, 2002 to 2015

an increasing number of mobile-only households with the majority of these types of telephone numbers not being listed [39]. However, studies have shown that using this sample frame is still a viable source and reliable estimates can be produced when applying more effective weighting techniques, such as raked weighting [10], to overcome the sampling bias as well as non-response bias.
A further weakness is the lack of power in terms of data on Aboriginal status. In Australia, recent policy actions have focused on improving the health of Aboriginal populations with the Prime Minister of Australia in 2008 signing a statement committed to developing a long-term plan of action to end health inequalities between indigenous and non-indigenous populations [32].

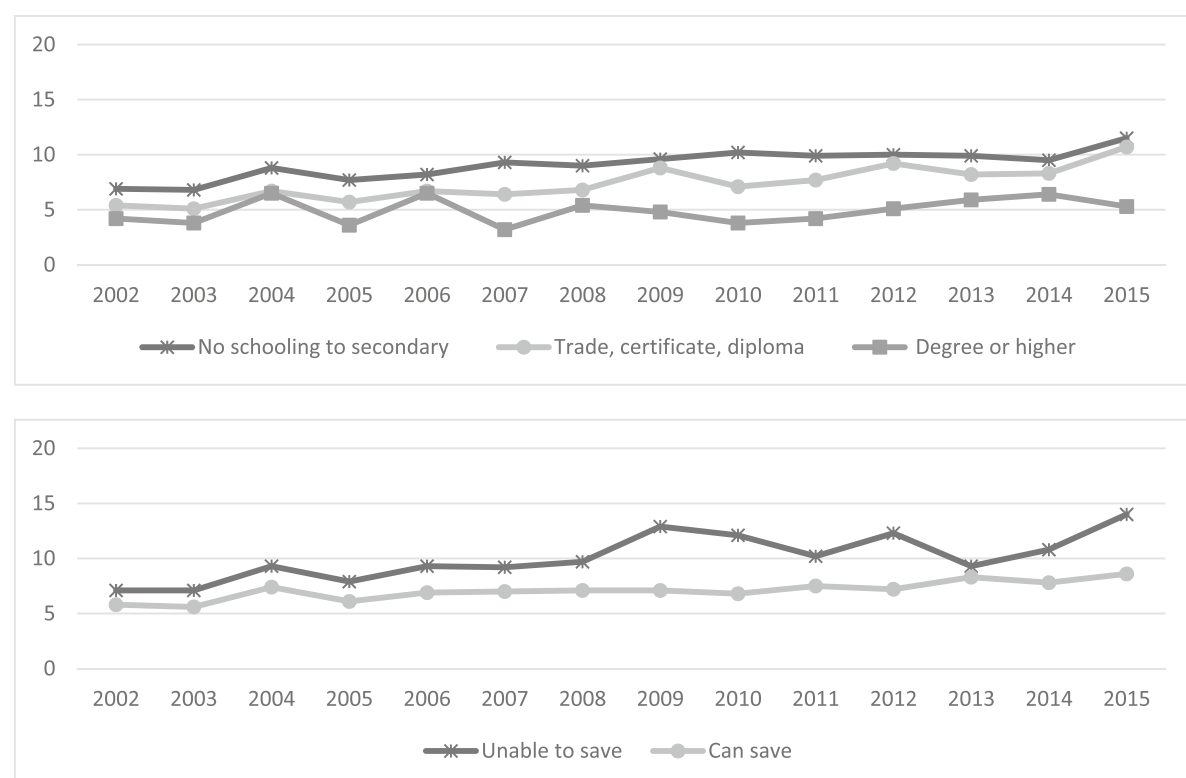

Fig. 4 Annual prevalence, diagnosed diabetes by educational attainment and household money situation, 2002 to 2015 


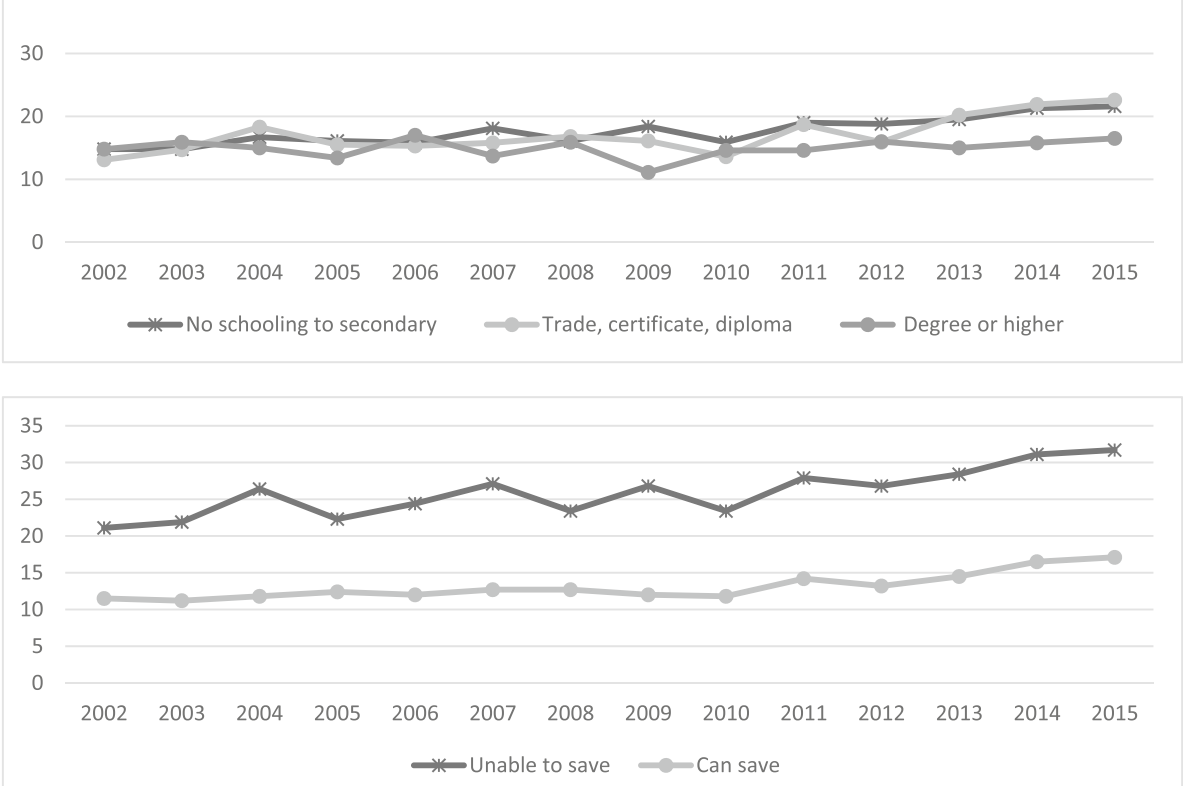

Fig. 5 Annual prevalence, diagnosed mental health condition by educational attainment and household money situation, 2002 to 2015

Although SAMSS collects this information, the limited sample size does not permit analysis by Aboriginal status. It is also acknowledged that some of the increases in prevalence of mental health problems reported in this analysis could be the result of better diagnosing, which has been supported by additional funding from the
Federal government in recent years. This could also impact our analyses by social class, leading to an underestimation of inequalities, since individuals who are more educated are also more likely to seek health care services and receive a diagnosis. The acknowledgment of public health campaigns in increasing the fruit consumption has
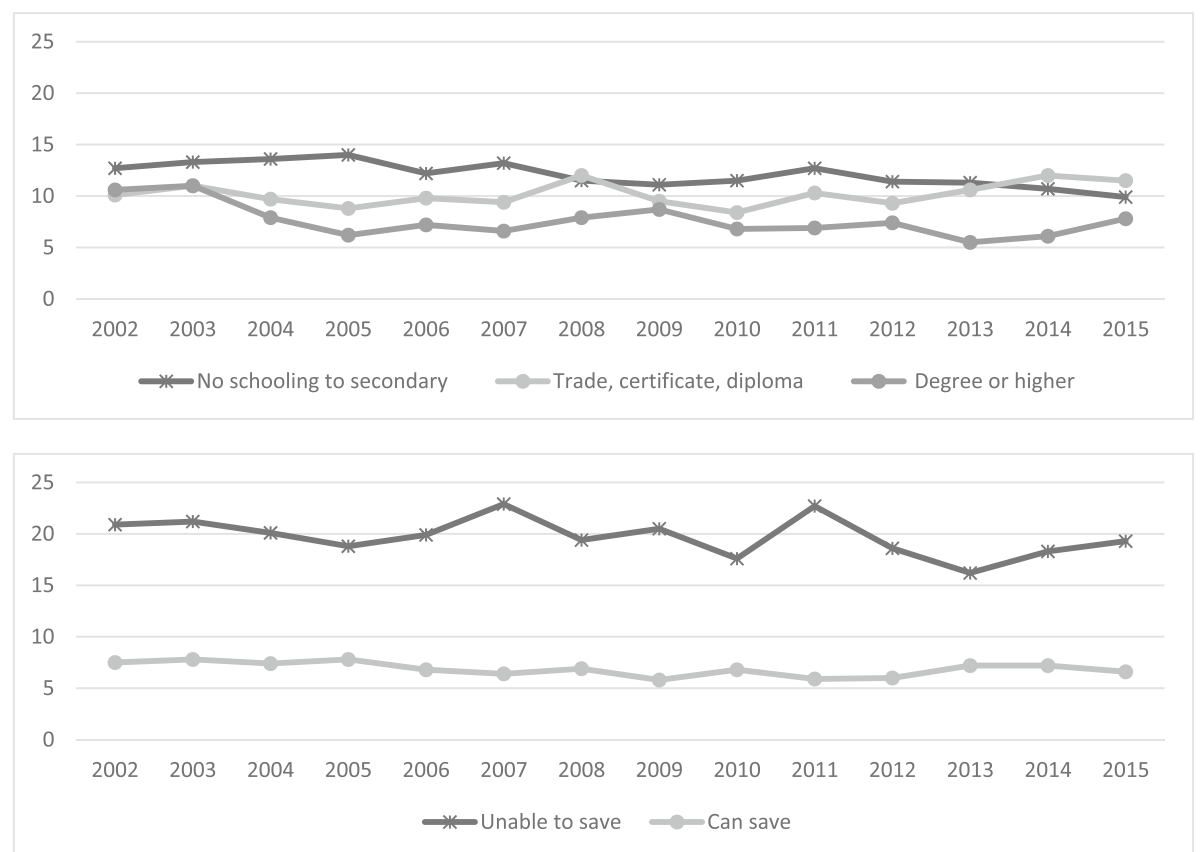

Fig. 6 Annual prevalence, psychological distress by educational attainment and household money situation, 2002 to 2015 

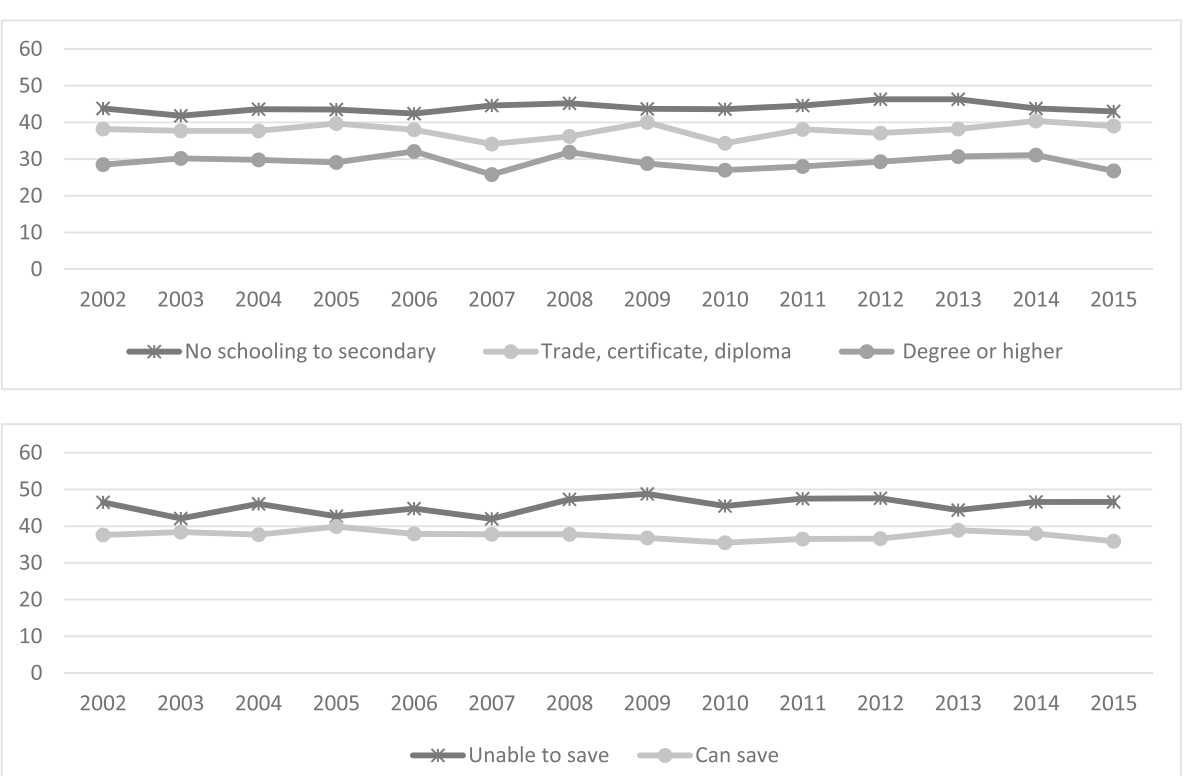

Fig. 7 Annual prevalence, at least one chronic condition by educational attainment and household money situation, 2002 to 2015

also not been fully explored although earlier work with this surveillance system has shown promising results [35].

\section{Conclusions}

It is evident, and this paper has contributed to providing more evidence, that there is much work still to do to "close the gap" [28, 40]. Also in more egalitarian societies with universal health systems, such as SA, more targeted and effective interventions are evidently needed to change the trends highlighted by SAMSS. BRFS can be a good source of information both to show the evolution of problems and to evaluate possible future interventions. Much effort is still required to 'close the gap' of health inequalities in SA. More precisely targeted and properly implemented interventions are needed.

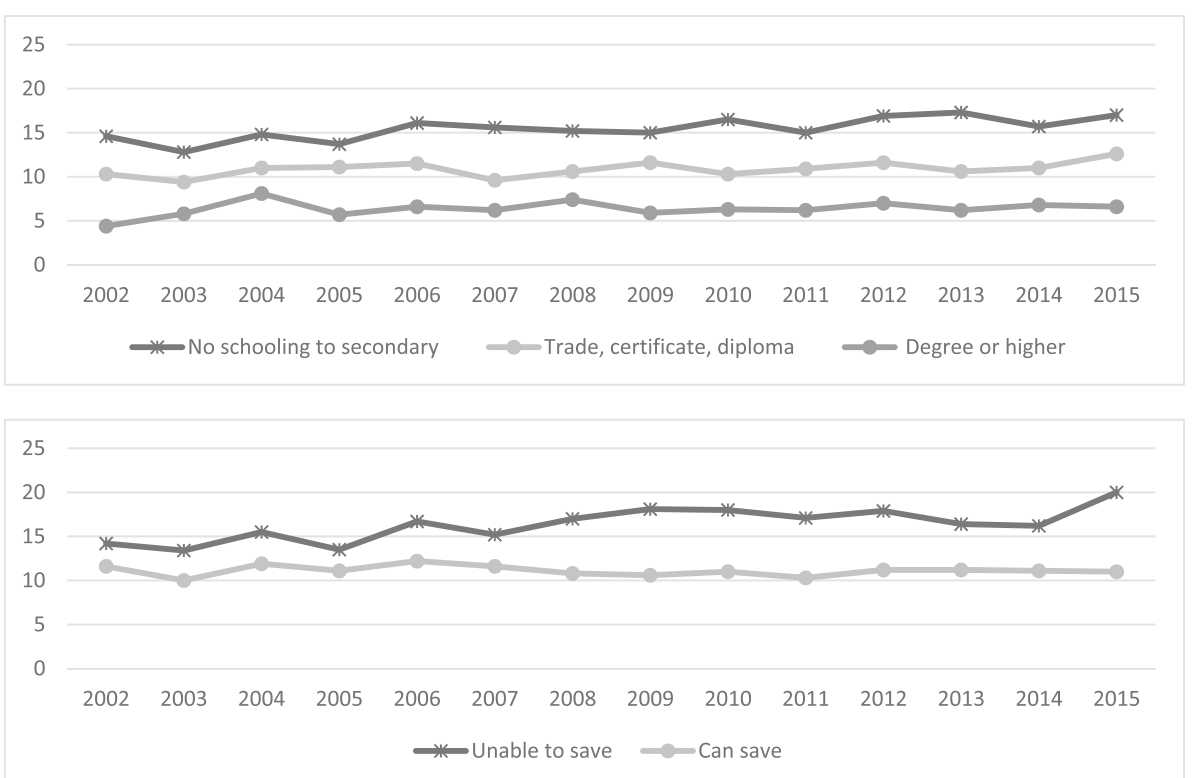

Fig. 8 Annual prevalence, two or more chronic conditions by educational attainment and household money situation, 2002 to 2015 


\section{Additional file}

Additional file 1: Table S1. Total sample size and median response rates, 2002 to 2015. Note: Response rates (RR1) were calculated from the final dispositions of the telephone numbers using the American Association for Public Opinion Research (AAPOR) [18] standard definitions. (DOCX 12 kb)

\section{Abbreviations}

AAPOR RR1: American Association for Public Opinion Research Response Rate \#1; ABS: Australian Bureau of statistics; BMl: Body mass index; CATI: Computer assisted telephone interviewing; EWP: Electronic white pages; HREC: Human research ethics committee; K10: Kessler 10; PROS: Population research \& outcome studies; SA: South Australia; SAH: SA health; SAMSS: South Australian monitoring \& surveillance system; WHO: World health organization

\section{Acknowledgments}

SAMSS is owned by Department of Health and Ageing, SA, Australia. All collected source data were maintained and managed by Population Research and Outcome Studies, University of Adelaide until March 2017. The opinions expressed in this work are those of the authors and may not represent the position or policy of Department for Health and Ageing.

\section{Funding}

Not applicable.

\section{Availability of data and materials}

Data are available on request from the HREC, SA Health.

\section{Authors' contributions}

SC \& AWT designed the study, SC wrote the original draft, EDG analyzed the data. All authors reviewed, edited and approved the final manuscript.

\section{Ethics approval and consent to participate}

The SAMSS methodology questionnaire has been approved by the South Australian Department of Health Ethics of Human Research Committee ((HREC/14/SAH/200 \& HREC/436.02.2014). Consent to participate was implied by non-response to letter of introduction with an opt-out number to call, acceptance of the telephone call and continuation of the survey and was approved by the Ethics committee.

\section{Consent for publication}

Not applicable.

\section{Competing interests}

The authors declare that they have no competing interests.

\section{Publisher's Note}

Springer Nature remains neutral with regard to jurisdictional claims in published maps and institutional affiliations.

\section{Author details}

'Department of Economics, Ca' Foscari University of Venice, San Giobbe 873, 30121 Venice, Italy. ${ }^{2}$ School of Medicine, The University of Adelaide, Adelaide, South Australia. ${ }^{3}$ Population Research \& Outcome Studies, Discipline of Medicine, The University of Adelaide, Adelaide, South Australia.

Received: 18 June 2018 Accepted: 14 December 2018 Published online: 08 January 2019

\section{References}

1. Popham F, Dibben C, Bambra C. Are health inequalities really not the smallest in the Nordic welfare states? A comparison of mortality inequality in 37 countries. J Epidemiol Community Health. 2013;67(5):412-8.

2. Department of Human Services. Better Health Outcomes for Australians: National Goals, Targets and Strategies for Better Health Outcomes into the Next Century. Canberra: Australia Commonwealth; 1994.
3. Draper G, Turrell G, Oldenburg B. Health Inequalities in Australia: Mortality. Monitoring Series No. 1. AlHW Cat No PHE 55. Canberra: Queensland University of Technology \& the Australian Institute of Health and Welfare; 2004.

4. Turrell G, Mathers CD. Socioeconomic status and health in Australia. Med J Aust. 2000;172(9):434-8.

5. Turrell G, Stanley L, de Looper M, Oldenburg B. Health Inequalities in Australia: Morbidity, Health Behaviours, Risk Factors and Health Service Use. Health Inequalities Monitoring Series NO 2. AlHW Cat No. PHE 72. Canberra: Queensland University of Technology \& the Australian Institute of Health \& Welfare; 2006

6. WHO. 2010. Adelaide statement on Health in all policies. Adelaide: WHO, government of South Australia. Downloaded from: https://www.who.int/ social_determinants/hiap_statement_who_sa_final.pdf.

7. SA Health. 2017. Protect, prevent, improve, inform - the chief public Health Officer's report 2014-2016, South Australian Government, Adelaide, South Australia. ISBN 978-1-74243-849-8. Available from https://www.sahealth.sa. gov.au/wps/wcm/connect/35b7bc804102b6f19fa4df1afc50ebfc/16149+Chief +Public+Health+2016+Report-FINAL.pdf?MOD=AJPERES.

8. WHO - Non-communicable Diseases (NDC) Country Profiles, 2018 Australia. Available from: https://www.who.int/nmh/countries/aus_en. pdf?ua $=1$.

9. Marmot M, Goldblatt P. Importance of monitoring health inequalities. $\mathrm{Br}$ Med J. 2013;347:f6576.

10. Taylor AW, Campostrini S, Gill TK, Herriot M, Carter P, Dal Grande E. The use of chronic disease risk factor surveillance systems for evidence-based decision-making - physical activity and nutrition as examples. Int J Public Health. 2010;55(4):243-9.

11. Population Research \& Outcome Studies (PROS). South Australian Monitoring and Surveillance System (SAMSS) technical report. Adelaide: SA Department of Health; 2004

12. Dal Grande E, Chittleborough CR, Campostrini S, Tucker G, Taylor AW. Health estimates using survey raked-weighting techniques in an Australian population health surveillance system. Am J Epidemiol. 2015;182(6):544-56.

13. National Health and Medical Research Council. 2005. The dietary guidelines for Australians. ISBN 1864961171

14. WHO. Diet, nutrition and the prevention of chronic diseases: report of a Joint WHO/FAO Expert Consultation. WHO technical report series, no. 916. Geneva: World Health Organization; 2003.

15. Raphael D, Anstice S, Raine K, McGannon KR, Kamil Rizvi S, Yu V. The social determinants of the incidence and management of type 2 diabetes mellitus: are we prepared to rethink our questions and redirect our research activities? Leadersh Health Serv. 2003;16(3):10-20.

16. Marmot M, Wilkinson R, editors. Social determinants of health. Oxford: Oxford University Press; 2005.

17. Population Research and Outcome Studies (PROS). 2002. The Kessler Psychological Distress Scale (K10). Adelaide: Department of Health; 2002. Report No: 14.

18. Australian Bureau of Statistics (ABS). National Survey of Mental Health and Wellbeing: Summary of Results. Canberra: Australian Bureau of Statistics. Report No: 4326.0; 2007.

19. Andrews G, Slade T. Interpreting scores on the Kessler psychological distress scale (K10). Aust N Z J Public Health. 2001;25(6):494-7.

20. The American Association for Public Opinion Research. Standard definitions: final dispositions of case codes and outcome rates for surveys. Deerfield Illinois: AAPOR; 2011.

21. Australian Bureau of Statistics (ABS), Household Income and Wealth Australia, Report No: 6523.0. http://www.abs.gov.au/AUSSTATS/abs@.nsf/ DetailsPage/6523.02015-16. Accessed 25 Apr 2016.

22. Mackenbach JP. The persistence of health inequalities in modern welfare states: the explanation of a paradox. Soc Sci Med. 2012;75(4):761-9.

23. Frohlich $\mathrm{KL}$, Potvin $\mathrm{L}$. Transcending the known in public health practice: the inequality paradox: the population approach and vulnerable populations. Am J Public Health. 2008;98(2):216-21.

24. Barr DA. 2014. Health disparities in the United States: Social class, race, ethnicity, and health. John Hopkins University press. ISBN-13 978-0801888212.

25. Kreuter MW, Hovmand P, Pfeiffer DJ, Fairchild M, Rath S, Golla B, Casey C. The "long tail" and public Health: new thinking for addressing Health disparities. Am J Public Health. 2004:104(12):2271-8.

26. Campostrini S. 2013. Surveillance for NCDs and Health Promotion: An Issue of Theory and Method in global handbook on noncommunicable diseases and Health promotion (Ed McQueen DV), Springer. ISBN 978-1-4614-7594-1. 
27. Campostrini S, McQueen D, Taylor A, Daly A. World Alliance for risk factor surveillance white paper on surveillance and Health promotion. AIMS Public Health. 2015;2:10-26.

28. WHO. NCD Surveillance strategy. https://www.who.int/ncd_surveillance/ strategy/en/. Accessed 25 Apr 2016.

29. Campostrini S, McQueen DV. Inequalities: the "gap" remains; can surveillance aid in closing the gap? Int J Public Health. 2014;59:1-2.

30. Assaf D, Campostrini S, Di Novi C, Xu F, Gotway Crawford C. Analyzing disparity trends for health care insurance coverage among non-elderly adults in the US: evidence from the behavioral risk factor surveillance system, 1993-2009. Eur J Health Econ. 2017:18:387-98.

31. Assaf D, Campostrini S, Xu F, Gotway Crawford C. Analysing behavioural risk factor surveillance data by using spatially and temporally varying coefficient models. J R Stat Soc Series A Stat Soc. 2016;179:153-75.

32. Australian Government. Indigenous Health Equality Summit Statement of Intent. Canberra: Australian Government; 2008. Downloaded from: https:// www.humanrights.gov.au/publications/close-gap-indigenous-healthequality-summit-statement-intent

33. Abel T. Cultural capital and social inequality in health. J Epidemiol Community Health. 2008;62(7):e13.

34. Bernard P, Charafeddine R, Frohlich KL, Daniel M, Kestens Y, Potvin L. Health inequalities and place: a theoretical conception of neighbourhood. Soc Sci Med. 2007;65(9):1839-52

35. Campostrini S, Holtzman D, McQueen DV, Boaretto E. Evaluating the effectiveness of Health promotion policy: changes in the law on drinking and driving in California. Health Promot Int. 2006:21:130-5.

36. Mackenbach JP. Can we reduce health inequalities? An analysis of the English strategy (1997-2010). J Epidemiol Community Health. 2011;65:568-75.

37. CATI Technical Reference Group. Field test 1 report: asthma, demographic characteristics and diabetes. Canberra: Australian Institute of Health and Welfare; 2003.

38. Taylor AW, Dal Grande E, Gill TK, Chittleborough CR, Wilson DH, Adams RJ, Grant JF, Phillips P, Appleton S, Ruffin RE. How valid are self-reported height and weight? A comparison between CATI self-report and clinic measurements using a large cohort study. Aust N Z J Public Health. 2006;30:238-46.

39. Dal Grande E, Chittleborough CR, Campostrini S, Taylor AW. Bias of health estimates obtained from chronic disease and risk factor surveillance systems using telephone population surveys in Australia. Results from a representative face-to-face survey in Australia from 2010 to 2013. BMC Med Res Methodol. 2016;16:44.

40. Marmot M, Friel S, Bell R, Houweling TA, Taylor S. Commission on social determinants of Health. Closing the gap in a generation: health equity through action on the social determinants of health. Lancet. 2008;372(9650): $1661-9$

Ready to submit your research? Choose BMC and benefit from:

- fast, convenient online submission

- thorough peer review by experienced researchers in your field

- rapid publication on acceptance

- support for research data, including large and complex data types

- gold Open Access which fosters wider collaboration and increased citations

- maximum visibility for your research: over $100 \mathrm{M}$ website views per year

At $\mathrm{BMC}$, research is always in progress.

Learn more biomedcentral.com/submissions 